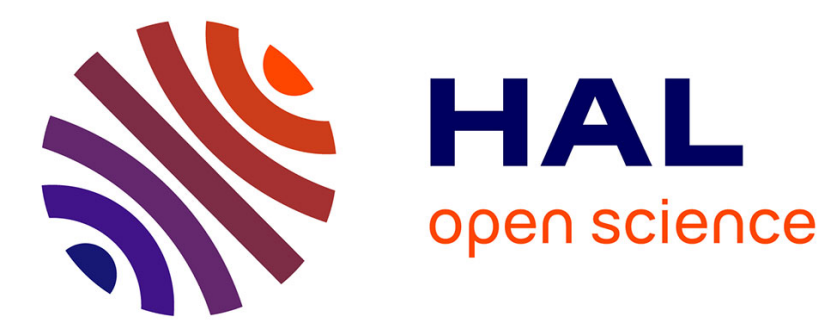

\title{
Stochastic hyperelastic constitutive laws and identification procedure for soft biological tissues with intrinsic variability \\ B Staber, J Guilleminot
}

\section{- To cite this version:}

B Staber, J Guilleminot. Stochastic hyperelastic constitutive laws and identification procedure for soft biological tissues with intrinsic variability. Journal of the mechanical behavior of biomedical materials, 2017, 65, pp.743-752. 10.1016/j.jmbbm.2016.09.022 . hal-01367381

\section{HAL Id: hal-01367381 \\ https://hal.science/hal-01367381}

Submitted on 16 Sep 2016

HAL is a multi-disciplinary open access archive for the deposit and dissemination of scientific research documents, whether they are published or not. The documents may come from teaching and research institutions in France or abroad, or from public or private research centers.
L'archive ouverte pluridisciplinaire HAL, est destinée au dépôt et à la diffusion de documents scientifiques de niveau recherche, publiés ou non, émanant des établissements d'enseignement et de recherche français ou étrangers, des laboratoires publics ou privés. 


\title{
Stochastic hyperelastic constitutive laws and identification procedure for soft biological tissues with intrinsic variability
}

\author{
B. Staber, J. Guilleminot* \\ Université Paris-Est, Laboratoire Modélisation et Simulation Multi Echelle, MSME UMR 8208 CNRS, 5 bd Descartes, 77454 \\ Marne-la-Vallée, France
}

\begin{abstract}
In this work, we address the constitutive modeling, in a probabilistic framework, of the hyperelastic response of soft biological tissues. The aim is on the one hand to mimic the mean behavior and variability that are typically encountered in the experimental characterization of such materials, and on the other hand to derive mathematical models that are almost surely consistent with the theory of nonlinear elasticity. Towards this goal, we invoke information theory and discuss a stochastic model relying on a low-dimensional parametrization. We subsequently propose a two-step methodology allowing for the calibration of the model using standard data, such as mean and standard deviation values along a given loading path. The framework is finally applied and benchmarked on three experimental databases proposed elsewhere in the literature. It is shown that the stochastic model allows experiments to be accurately reproduced, regardless of the tissue under consideration.
\end{abstract}

This is the preprint version of the following article: Stochastic hyperelastic constitutive laws and identification procedure for soft biological tissues with intrinsic variability, which has been accepted for publication in the Journal of the Mechanical Behavior of Biomedical Materials (DOI 10.1016/j.jmbbm.2016.09.022). This manuscript version is made available under the CC-BY-NC-ND 4.0 license.

Keywords: biological material, calibration, constitutive law, hyperelasticity, information theory, variability 2010 MSC: 74A40, 74B20, 74Q15, 74S60

\section{Preliminaries}

\subsection{Introduction}

The constitutive modeling and data-driven calibration of the mechanical behavior of soft biological tissues is of 5 paramount importance in a wide range of applications from bioengineering and computational - and potentially, multiscale - mechanics of hierarchical biomaterials (see [9. 13, 12] for the modeling of arteries, for instance) to computed-assisted surgery (CAS). While the definition of 10 suitable models is still the matter of active research, due to the great complexity and multiphysics aspects of the mechanisms governing the macroscopic behavior, one additional difficulty lies in the fact that the experimental characterization of such materials typically exhibits a large variability; see, in a non-exhaustive manner, [22, 23, 43, [14, 29, 32, 17, 30, 31, 35, 6, 33, 34, 39] and [42 for experimental characterizations of brain tissues, liver tissues, spinal cord white matter and abdominal organs, respectively. These uncertainties, which may be due to various

\footnotetext{
* Corresponding author

Email address: johann.guilleminot@u-pem.fr (J. Guilleminot)
} subject variability, age, gender or even potential disease), may critically impact simulation predictiveness or clinical diagnosis for example.

In practice, these fluctuations are usually ignored and smoothed out through an averaging procedure along the statistical dimension. This mean response is subsequently fitted by solving a least-squares problem by means of a deterministic optimization method (see 28). Whereas such approximations may be found satisfactory in some cases, depending on the application under consideration, performing high-fidelity computations or making informed decisions requires the modeling and calibration issues to be both addressed in a probabilistic framework. From a stochastic standpoint, the Bayesian paradigm provides a sound approach to data-driven modeling for deterministic and random parameters 4, 10. This natural path was very recently pursued in [21] and previously used by the same authors for model selection in 20.

In this work, we propose an alternative methodology of randomized parameters are constructed explicitly. The 
main advantage of such a formulation (in contrast to a Bayesian approach where the posterior distributions are generally unknown analytically) is that it allows existence results for the solution of the associated boundary value problem to be invoked (with probability one). In practice, ${ }^{90}$ the proposed class of stochastic stored energy functions can purposely be used as:

- an admissible model making the identification task well-posed (from a mechanical standpoint) whenever experimental data are limited;

- a prior model in a Bayesian setting, especially when ${ }^{95}$ the number of the parameters defining the model (which are referred to as hyperparameters) is large; see [37] for an example in three-dimensional linear elasticity.

The aim of this research is twofold. First, it attempts to lay out a framework, based on information theory, for the modeling of stochastic stored energy functions. To this purpose, we provide a self-contained and applicationoriented presentation of a stochastic model derived for the Ogden class of stored energy functions [40, 41]. This class turns out to be relevant to the modeling of some isotropic biological materials (see [5] for example). Additionally, we address the definition of a calibration methodology. The latter specifically allows for the identification of the model hyperparameters using mean and variance estimates along a loading curve. Second, it aims at assessing the capability of these models to reproduce the experimental variability 70 that is observed in the mechanical response of biological tissues at large strains (and small strain rates). From a clinical perspective, these statistical fluctuations may be considered as informative quantities in the early stage prognosis of diseases affecting the mechanical response of the tissues under consideration.

This outline of this paper is as follows. In Section 2 , we first set up notation and provide a brief reminder on deterministic constitutive modeling in hyperelasticity. The information-theoretic stochastic model and the calibration procedure are then presented in Section 3. Finally, some applications involving experimental results taken from the literature are discussed in Section 4

\subsection{Notation}

Throughout this paper, deterministic scalars, vectors and second-order tensors are denoted by $a, \boldsymbol{a}$ and $[A]$. Their stochastic counterparts are respectively denoted by $A, \boldsymbol{A}$ and $[\boldsymbol{A}]$. The table of main notations is reported below (here, stresses are defined for a uniaxial compression test).

$\begin{array}{ll}w & \text { Deterministic stored energy function } \\ W & \text { Random stored energy function } \\ \mu & \text { Deterministic shear modulus } \\ \mu & \text { Random shear modulus } \\ \sigma & \text { Principal first Piola-Kirchoff stress }\end{array}$

$\Sigma \quad$ Random principal first Piola-Kirchoff stress

$\mathbb{E}\{\cdot\} \quad$ Mathematical expectation

$\|[A]\| \quad$ Frobenius norm of $[A]$

Finally, $k_{0}$ denotes a normalization constant, the value of which may change from line to line.

\section{Deterministic constitutive model}

\subsection{Basic notation and kinematics}

Let $\Omega$ be the region in $\mathbb{R}^{3}$ occupied by the body under consideration at rest and let $\partial \Omega$ denote its boundary. The body is assumed to undergo a deformation $\boldsymbol{\varphi}: \Omega \ni \boldsymbol{x} \mapsto$ $\boldsymbol{x}^{\varphi} \in \Omega^{\varphi}$, where $\Omega^{\varphi}$ is the current configuration. The mapping $\varphi$ is classically assumed to be sufficiently smooth, orientation-preserving and injective.

The associated deformation gradient $[F]$ is defined as

$$
[F(x)]=\nabla_{x} \varphi(x), \quad \forall x \in \Omega,
$$

and satisfies the condition $\operatorname{det}([F])>0$ in $\bar{\Omega}$. For the sake of notational convenience, spatial indexing is omitted from now on. The principal stretches of the deformation gradient are denoted as $\left\{v_{j}([F])\right\}_{j=1}^{3}$ (or simply $\left\{v_{j}\right\}_{j=1}^{3}$ when no confusion can occur), and the cofactor matrix $\operatorname{Cof}([F])$ of $[F]$ is defined as

$$
\operatorname{Cof}([F])=\operatorname{det}([F])[F]^{-\mathrm{T}} .
$$

\subsection{Constitutive equation}

In this work, we consider homogeneous incompressible hyperelastic materials defined by a stored energy function $w$ such that

$$
[T]=\frac{\partial w([F])}{\partial[F]}-\varpi[F]^{-\mathrm{T}},
$$

where $[T]$ is the first Piola-Kirchhoff stress tensor (which is also referred to as the engineering stress), $[F]$ denotes the deformation gradient (with unitary determinant) and $\varpi$ is a Lagrange multiplier raised by the incompressibility condition. Additionally, we focus on Ogden-type stored energy functions for isotropic hyperelastic materials. Such functions are defined, at orders $m \geqslant 1$ and $n \geqslant 1$, as [27]

$$
w([F])=\sum_{i=1}^{m} p_{i} \Phi_{\eta_{i}}([F])+\sum_{j=m+1}^{m+n} p_{j} \Upsilon_{\eta_{j}}([F]),
$$

with

$$
\Phi_{\eta_{i}}([F])=v_{1}^{\eta_{i}}+v_{2}^{\eta_{i}}+v_{3}^{\eta_{i}}-3
$$

(with a slight abuse of notation) and

$$
\Upsilon_{\eta_{j}}([F])=\left(v_{1} v_{2}\right)^{\eta_{j}}+\left(v_{1} v_{3}\right)^{\eta_{j}}+\left(v_{2} v_{3}\right)^{\eta_{j}}-3,
$$

where it is recalled that $\left\{v_{j}\right\}_{j=1}^{3}$ are the principal stretches associated with the deformation gradient $[F]$.

Upon expanding the stored energy function near the reference configuration, it is found that the parameters 
$\boldsymbol{p}=\left(p_{1}, \ldots, p_{n_{p}}\right)$ and $\boldsymbol{\eta}=\left(\eta_{1}, \ldots, \eta_{n_{p}}\right)$ must satisfy the following additional consistency relation [27]:

$$
\sum_{k=1}^{n_{p}} p_{k} \eta_{k}^{2}=2 \mu
$$

where $\mu>0$ is the shear modulus.

The applications considered in this paper consist in simple compression tests along a principal direction, say $\boldsymbol{e}_{3}=(0,0,1)$. In this case, and by combining Eq. (3) and the incompressibility condition $v_{1} v_{2} v_{3}=1$, one can 130 show (see e.g. [27, 12]) that the resulting principal PiolaKirchhoff stress $\sigma$ writes

$$
\begin{aligned}
\sigma(v)= & \sum_{i=1}^{m} p_{i} \eta_{i}\left(v^{\eta_{i}-1}-v^{-1-\eta_{i} / 2}\right) \\
& -\sum_{j=m+1}^{m+n} p_{j} \eta_{j}\left(v^{-\eta_{j}-1}-v^{-1+\eta_{j} / 2}\right)
\end{aligned}
$$

where the substitution $v=v_{3}$ has been introduced for notational convenience.

\subsection{Fundamental assumptions}

From a theoretical standpoint, the available existence theorems for boundary value problems in hyperelasticity (see the references hereinafter) rely on the existence of minimizers for the total potential energy function and were established by resorting on the calculus of variations. Not145 surprisingly, the investigation of sufficient conditions on the stored energy function such that the aforementioned existence results can effectively be proven has attracted much attention from researchers in applied mathematics and theoretical mechanics. To date, minimal requirements are essentially concerned with some generalized convexity ${ }^{150}$ conditions, as well as with a coerciveness inequality that models an asymptotic behavior at large strains; see the seminal work reported in [24], as well as the textbook [7]. Certainly one of the most invoked assumption is that of polyconvexity, introduced by Ball in [1. This fundamental property, when complemented by a set of suitable growth ${ }_{155}$ conditions, insures the existence of at least one minimizer for the total potential energy; hence ensuring the wellposedness of the nonlinear boundary value problem (see also 3 for a specific discussion on biological materials).

In what follows, and following the result by Müller et ${ }_{160}$ al. 25], we assume that (see [2])

$$
\begin{aligned}
& p_{k}>0, \quad 1 \leqslant k \leqslant n_{p}, \\
& \eta_{1} \geqslant \cdots \geqslant \eta_{m} \geqslant 1, \quad \eta_{1} \geqslant 2, \\
& \eta_{m+1} \geqslant \cdots \geqslant \eta_{n_{p}} \geqslant 1, \quad \eta_{m+1} \geqslant \frac{3}{2},
\end{aligned}
$$

with $n_{p}=m+n$, ensuring the existence of at least one minimizer for the total potential energy.
Remark 1. The single term stored energy function

$$
w([F])=p\left(v_{1}^{\eta}+v_{2}^{\eta}+v_{3}^{\eta}-3\right)
$$

is covered by the existence results if $p>0$ and $\eta \geqslant 3$ (see [1). Moreover, the consistency with linearized elasticity yields $p \eta^{2}=2 \mu$, so that the stored energy function writes:

$$
w([F])=\frac{2 \mu}{\eta^{2}}\left(v_{1}^{\eta}+v_{2}^{\eta}+v_{3}^{\eta}-3\right) .
$$

The particular case $\eta=2$ leads to the well known NeoHookean model in three-dimensional elasticity, which is not covered by the existence results invoked in this paper.

In the next section, we first discuss the randomization of Ogden-type stored energy functions. A new methodology allowing for the calibration of the probabilistic model is subsequently proposed.

\section{Stochastic hyperelastic constitutive models}

The generalization of hyperelastic constitutive laws in a stochastic context has received little attention to date. The main difficulty raised by such a generalization stems from the fact that the stochastic stored energy functions are simultaneously required (i) to accurately reproduce the experimental trends, and (ii) to be consistent with the theory of nonlinear elasticity (see Section 2.3). It should be noticed that considering one single constraint apart from the other one may lead to models that

- fit dispersed data very well (in terms of mean and variance at a given stretch for example), but are not consistent with theoretical aspects - hence yielding non-admissible samples for subsequent analysis;

- are fully consistent with existence theorems, but may not satisfactorily reproduce observed experimental results.

Clearly, any of the above situations reduces the predictive capabilities of the associated modeling framework. Note in addition that experimental data are often very limited, so that the direct computation of statistical estimators at a reasonable level of convergence is itself an issue - in this case, the use of the maximum likelihood method may alternatively be considered.

Very recently, new contributions accommodating the aforementioned modeling constraints in the framework of information theory [15, 16] were proposed; see [40] for the case of incompressible materials, as well as 41 for the compressible case. In these works, stochastic stored energy functions are constructed by randomizing, in a proper way, some parameters involved in the definition of classical, e.g. Ogden-type stored energy functions. More specifically, and following the maximum entropy (MaxEnt) principle, the probability density functions defining these random parameters are constructed by maximizing the so-called 
Shannon differential entropy 36 over the set of probability density functions that satisfy a set of contraint equations (see Appendix A for more details). The latter, which are expressed in the form of mathematical expectations, are essentially induced by the information that is available on the set of random model parameters. This information is typically inferred from the properties ensuring the well- ${ }^{20}$ posedness of the associated variational formulation, such as positiveness, or from known statistical moments. By construction, such an approach reduces the modeling bias, especially when compared to methodologies relying on the a priori choice of standard statistical distributions, and readily ensures that all sufficient mathematical conditions are fulfilled (in accordance with the results from Section 2.3. The main result derived in [40] is first presented for completeness in Section 3.1. A novel methodology allowing for the calibration of the stochastic stored energy function using typically available experimental results (namely, the mean and variance evolutions) is then proposed in Section 3.2. This calibration strategy will be tested for various biological tissues in Section 4 .

\subsection{Construction of stochastic Ogden-type stored energy functions}

Let $\boldsymbol{P}$ and $\mu$ be the random variables corresponding to the stochastic modeling of the vector-valued parameter $\boldsymbol{p}$ and the shear modulus $\mu$, respectively. The stochastic version $W$ of the Ogden potential $w$ is then written as

$$
W([F])=\sum_{i=1}^{m} P_{i} \Phi_{\eta_{i}}([F])+\sum_{j=m+1}^{m+n} P_{j} \Upsilon_{\eta_{j}}([F]),
$$

where $\Phi_{\eta_{i}}$ and $\Upsilon_{\eta_{j}}$ are defined by Eqs. (5)-(6), and the exponents $\left\{\eta_{k}\right\}_{k=1}^{n_{p}}$ are kept deterministic and such that the restrictions given by Eqs. (9b)- $(9 \mathrm{c})$ hold. The random variables $\boldsymbol{P}$ and $\mu$ also satisfy the consistency relation (see Eq. (7))

$$
\sum_{k=1}^{n_{p}} P_{k} \eta_{k}^{2}=2 \mu
$$

almost surely. It should be noticed that the stochastic models of $\boldsymbol{P}$ and $\boldsymbol{\mu}$ completely define the probabilistic model of the stochastic stored energy function $W$ for $\mathrm{a}^{205}$ given value of the deformation gradient $[F]$.

In a first step, the probability density function $p_{\mu}$ of the random shear modulus $\mu$ is constructed by taking into account that $\mu>0$ almost surely, the mean constraint

$$
\mathbb{E}\{\mu\}=\underline{\mu}, \quad \underline{\mu}>0,
$$

and the additional constraint:

$$
\mathbb{E}\{\log (\mu)\}=\nu, \quad|\nu|<+\infty .
$$

From a theoretical standpoint, Eq. (15) ensures that $\mu$ and $\mu^{-1}$ are both second-order random variables (see [11]). According to the MaxEnt principle (see Appendix A.2 for technical details), the random variable $\mu$ then follows a Gamma distribution:

$$
\mu \sim \mathrm{G}\left(\rho_{1}, \rho_{2}\right)
$$

where the parameters $\rho_{1}>0$ and $\rho_{2}>0$ are raised by the MaxEnt principle.

In a second step, we introduce for technical convenience an auxiliary random variable $\boldsymbol{U}=\left(U_{1}, \ldots, U_{n_{p}-1}\right)$ such that (see Eq. (13))

$$
P_{k}=\frac{2 \mu}{\eta_{k}^{2}} U_{k}, \quad 1 \leqslant k \leqslant n_{p}-1
$$

and

$$
P_{n_{p}}=\frac{2 \llbracket}{\eta_{n_{p}}^{2}}\left(1-\sum_{k=1}^{n_{p}-1} U_{k}\right) .
$$

By construction (see Eq. (9a)), the random variable $\boldsymbol{U}$ takes its values in the admissible set

$$
\mathcal{S}=\left\{\boldsymbol{u} \in(] 0,1[)^{n_{p}-1} \mid 1-\sum_{k=1}^{n_{p}-1} u_{k}>0\right\}
$$

that corresponds to the interior of a $\left(n_{p}-1\right)$-dimensional simplex. The probability density function $p_{\boldsymbol{U}}$ of $\boldsymbol{U}$ is derived by invoking the principle of maximum entropy under constraints that are induced by Eq. 9a (or equivalently, by Eq. (19); see [40]), namely:

$$
\begin{gathered}
\mathbb{E}\left\{\log \left(U_{k}\right)\right\}=\nu_{k}, \quad 1 \leqslant k \leqslant n_{p}-1, \\
\mathbb{E}\left\{\log \left(1-\sum_{k=1}^{n_{p}-1} U_{k}\right)\right\}=\nu_{n_{p}},
\end{gathered}
$$

where $\left|\nu_{k}\right|<+\infty$ for $1 \leqslant k \leqslant n_{p}$. It can then be shown (see Appendix A.3 that the random variable $\boldsymbol{U}$ follows a Dirichlet distribution:

$$
\boldsymbol{U} \sim \operatorname{Dir}\left(\lambda_{1}, \ldots, \lambda_{n_{p}}\right),
$$

where the strictly positive parameters $\left(\lambda_{1}, \ldots, \lambda_{n_{p}}\right)$ are the Lagrange multipliers that are introduced by the MaxEnt principle.

Note that the marginal probability distributions of $\boldsymbol{U}$ thus obtained are unimodal when $\lambda_{k}>1,1 \leqslant k \leqslant n_{p}$. Whenever required, the case of multimodal distributions can be handled, either by defining a mixture of the proposed unimodal distributions (provided that a one-to-one correspondence between the modes of the random variables can be identified), or by using other probabilistic representations, such as mixtures of polynomial chaos expansions (PCEs) 26] or PCEs defined with respect to multimodal probability distributions [38].

The main result derived in [40] then states that the stochastic stored energy function defined by Eq. (12), where

- $\boldsymbol{P}$ is defined by Eqs. $17-18$,

- $\mu \sim \mathrm{G}\left(\rho_{1}, \rho_{2}\right)$, with $\rho_{1}>0$ and $\rho_{2}>0$, 
- $\boldsymbol{U} \sim \operatorname{Dir}\left(\lambda_{1}, \ldots, \lambda_{n_{p}}\right)$ with $\lambda_{i}>0$ for $1 \leqslant i \leqslant n_{p}, \quad{ }_{245}$ is covered by the existence results (outlined in Section 2.3) 220 and consistent with linearized elasticity almost surely. It is therefore admissible almost surely, which in turn implies that the associated stochastic nonlinear boundary value problem admits at least one solution.

The stochastic constitutive law is finally defined as

$$
[\boldsymbol{T}]=\frac{\partial W([F])}{\partial[F]}-\varpi[F]^{-\mathrm{T}},
$$

where $[\boldsymbol{T}]$ denotes the random first Piola-Kirchhoff stress tensor. Using Eq. (8), it can be deduced that the random Piola-Kirchhoff stress $\Sigma$ for a simple tensile (or compression) test writes

$$
\begin{aligned}
\Sigma(v)= & \sum_{i=1}^{m} P_{i} \eta_{i}\left(v^{\eta_{i}-1}-v^{-1-\eta_{i} / 2}\right) \\
& -\sum_{j=m+1}^{m+n} P_{j} \eta_{j}\left(v^{-\eta_{j}-1}-v^{-1+\eta_{j} / 2}\right) .
\end{aligned}
$$

Remark 2. It is observed that the variance of the Piola-

Kirchhoff stress $\Sigma(v)$ is monotonically increasing with $v$. It follows that the proposed stochastic approach is indeed relevant to situations where the variance increases along the loading path (this feature being frequently encountered in practice). Nevertheless, some cases where the variance the experimental stress does not increase together with the principal stretch may be addressed by randomizing the model exponents $\left\{\eta_{k}\right\}_{k=1}^{n_{p}}$. While such a model could certainly be constructed by pursuing a similar informationtheoretic methodology, such a strategy would introduce a set of hyperparameters belonging to high-dimensional spaces - hence making the calibration step more intricate.

Remark 3. A robust generator for the random variable $\boldsymbol{U}$ can be obtained by introducing a set of Gamma random variables $\left\{Y_{i}\right\}_{i=1}^{n_{p}}$, with

$$
Y_{i} \sim \mathrm{G}\left(\lambda_{i}, 1\right)
$$

and by sampling $\boldsymbol{U}$ through the component-wise mapping (see [8])

$$
U_{i}:=Y_{i} \times\left(\sum_{k=1}^{n_{p}} Y_{k}\right)^{-1}, \quad 1 \leqslant i \leqslant n_{p}-1 .
$$

In the case $n_{p}=2, \boldsymbol{U}$ becomes a scalar random variable $U$ which follows a Beta distribution. The Matlab functions gamrnd and betarnd might be useful in order to generate samples of Gamma and Beta random variables.

\subsection{Identification of the stochastic stored energy functions based on experimental data: methodological aspects}

In this section, we propose a methodology aiming at the calibration of the model parameters, which are gathered in the vector-valued Lagrange multipliers $\boldsymbol{\lambda}=\left(\lambda_{1}, \ldots, \lambda_{n_{p}}\right)$ and $\boldsymbol{\rho}=\left(\rho_{1}, \rho_{2}\right)$, using standard experimental data.

More specifically, let $\left\{\xi_{k}^{\exp }\right\}_{k=1}^{N}$ and $\left\{\varsigma_{k}^{\exp }\right\}_{k=1}^{N}$ denote the sets of values for the mean and standard deviation of the random stress at prescribed stretches $\left\{v_{k}^{\exp }\right\}_{k=1}^{N}$. These sets define the experimental information based on which the stochastic representation has to be identified. For ease of explanation, we consider below the case of first-order stored energy functions $\left(m=n=1\right.$; hence $\left.n_{p}=2\right)$. The approach can easily be generalized to arbitrary orders at the expense of notational complexity. Closed-form expressions that are relevant to this case are first derived in Section 3.2.1. The two sequential steps involved in the calibration methodology are then described in Sections 3.2 .2 and 3.2 .3

\subsubsection{Closed-form relations for first-order stochastic stored energy functions}

When $m=n=1$, the random stored energy function writes

$$
\begin{aligned}
W([F])= & P_{1}\left(v_{1}^{\eta_{1}}+v_{2}^{\eta_{1}}+v_{3}^{\eta_{1}}-3\right) \\
& +P_{2}\left(\left(v_{1} v_{2}\right)^{\eta_{2}}+\left(v_{1} v_{3}\right)^{\eta_{2}}+\left(v_{2} v_{3}\right)^{\eta_{2}}-3\right),
\end{aligned}
$$

where the random variables $P_{1}$ and $P_{2}$ are given by

$$
P_{1}=\frac{2 \mu}{\eta_{1}^{2}} U, \quad P_{2}=\frac{2 \mu}{\eta_{2}^{2}}(1-U),
$$

with $\eta_{1} \geqslant 2$ and $\eta_{2} \geqslant 3 / 2$. It is recalled that $\mu$ follows a Gamma distribution with parameters $\left(\rho_{1}, \rho_{2}\right)$, and that the random variable $U$ follows a Dirichlet distribution with parameters $\left(\lambda_{1}, \lambda_{2}\right)$. According to Eq. (24), the associated random Piola-Kirchhoff stress $\Sigma$ is given, for a uniaxial tensile (or compression) test, by

$$
\Sigma(v)=P_{1} \Psi_{1}(v)+P_{2} \Psi_{2}(v),
$$

where the functions $\Psi_{1}$ and $\Psi_{2}$ are defined as

$$
\Psi_{1}(v)=\eta_{1}\left(v^{\eta_{1}-1}-v^{-1-\eta_{1} / 2}\right)
$$

and

$$
\Psi_{2}(v)=-\eta_{2}\left(v^{-\eta_{2}-1}-v^{-1+\eta_{2} / 2}\right) .
$$

Moreover, it is readily deduced from Eq. (29) that the variance of $\Sigma(v)$ is given by

$$
\begin{aligned}
\operatorname{Var}\{\Sigma(v)\}= & \operatorname{Var}\left\{P_{1}\right\} \Psi_{1}^{2}(v)+\operatorname{Var}\left\{P_{2}\right\} \Psi_{2}^{2}(v) \\
& +2 \operatorname{Cov}\left\{P_{1}, P_{2}\right\} \Psi_{1}(v) \Psi_{2}(v),
\end{aligned}
$$

where $\operatorname{Var}\left\{P_{j}\right\}$ and $\operatorname{Cov}\left\{P_{1}, P_{2}\right\}$ denote the variance of $P_{j}$ and the covariance between $P_{1}$ and $P_{2}$, respectively. Next, and upon using the probabilistic properties of $\mu$ and $U$, it is found that:

$$
\operatorname{Var}\left\{P_{1}\right\}=\frac{4 \rho_{1} \rho_{2}^{2}}{\eta_{1}^{4}} \frac{\lambda_{1}\left(\lambda_{1}+\lambda_{2}+\lambda_{1} \lambda_{2}+\lambda_{1}^{2}+\rho_{1} \lambda_{2}\right)}{\left(\lambda_{1}+\lambda_{2}\right)^{2}\left(1+\lambda_{1}+\lambda_{2}\right)},
$$




$$
\operatorname{Var}\left\{P_{2}\right\}=\frac{4 \rho_{1} \rho_{2}^{2}}{\eta_{2}^{4}} \frac{\lambda_{2}\left(\lambda_{1}+\lambda_{2}+\lambda_{1} \lambda_{2}+\lambda_{2}^{2}+\rho_{1} \lambda_{1}\right)}{\left(\lambda_{1}+\lambda_{2}\right)^{2}\left(1+\lambda_{1}+\lambda_{2}\right)},
$$

and

$$
\operatorname{Cov}\left\{P_{1}, P_{2}\right\}=\frac{4 \rho_{1} \rho_{2}^{2}}{\eta_{1}^{2} \eta_{2}^{2}} \frac{\lambda_{1} \lambda_{2}\left(\lambda_{1}+\lambda_{2}-\rho_{1}\right)}{\left(\lambda_{1}+\lambda_{2}\right)^{2}\left(1+\lambda_{1}+\lambda_{2}\right)} .
$$

Substituting Eqs. (33), (34) and (35) into Eq. 32 allows us to estimate the variance of the stress $\Sigma(v)$ for given values of the hyperparameters (without resorting to Monte Carlo simulations).

\subsubsection{Defining Step 1: calibration of the mean model}

In the first step of the approach, which is formulated deterministically, the mean value

$$
\underline{\boldsymbol{p}}=\left(\underline{p}_{1}, \underline{p}_{2}\right):=\mathbb{E}\{\boldsymbol{P}\}
$$

of the random variable $\boldsymbol{P}$ is identified, in a classical manner 28, by fitting the mean experimental response with an Ogden-type constitutive model of orders $m=n=1$ :

$$
(\underline{\boldsymbol{p}}, \boldsymbol{\eta}):=\underset{(\boldsymbol{a}, \boldsymbol{b}) \in \mathbb{A} \times \mathbb{B}}{\operatorname{argmin}} \mathcal{J}(\boldsymbol{a}, \boldsymbol{b}),
$$

where the cost function $\mathcal{J}$ is given by

$$
\mathcal{J}(\boldsymbol{a}, \boldsymbol{b}):=\sum_{k=1}^{N}\left(\sigma\left(v_{k}^{\exp } ; \boldsymbol{a}, \boldsymbol{b}\right)-\underline{\xi}_{k}^{\exp }\right)^{2},
$$

and $\sigma\left(v_{k}^{\exp } ; \boldsymbol{a}, \boldsymbol{b}\right)$ is the predicted mean stress at the stretch $v_{k}^{\exp }$, obtained by substituting $(\boldsymbol{a}, \boldsymbol{b})$ for $(\boldsymbol{p}, \boldsymbol{\eta})$ in Eq. (8) (with $m=n=1$ ). The solution search is performed over the admissibles sets

$$
\mathbb{A}=\left(\mathbb{R}_{+}^{*}\right)^{2}
$$

and

$$
\mathbb{B}=\left\{\boldsymbol{\eta} \in \mathbb{R}^{2}, \quad \eta_{1} \geqslant 2, \quad \eta_{2} \geqslant 3 / 2\right\}
$$

induced by Eqs. 9a)-9c). This optimization problem can be solved by using a standard algorithm for constrained nonlinear problems.

3.2.3. Defining Step 2: calibration of $\left(\rho_{1}, \rho_{2}\right)$ and $\left(\lambda_{1}, \lambda_{2}\right)^{275}$

Let us address the calibration of the remaining hyperparameters $\left(\rho_{1}, \rho_{2}\right)$ and $\left(\lambda_{1}, \lambda_{2}\right)$. From Eq. (28) and the statistical independence of $\mu$ and $P_{j}$, we deduce that

$$
\underline{p}_{1}=\frac{2 \mathbb{E}\{\mu\}}{\eta_{1}^{2}} \mathbb{E}\{U\}
$$

and

$$
\underline{p}_{2}=\frac{2 \mathbb{E}\{\mu\}}{\eta_{2}^{2}}(1-\mathbb{E}\{U\}) .
$$

Since $\mu \sim \mathrm{G}\left(\rho_{1}, \rho_{2}\right)$ and $U \sim \operatorname{Dir}\left(\lambda_{1}, \lambda_{2}\right)$ (note that the ${ }^{285}$ Dirichlet distribution corresponds to the Beta distribution for univariate random variables), one has

$$
\underline{\mu}=\rho_{1} \rho_{2}
$$

and

$$
\underline{p}_{1}=\frac{2 \underline{\mu}}{\eta_{1}^{2}} \frac{\lambda_{1}}{\lambda_{1}+\lambda_{2}}, \quad \underline{p}_{2}=\frac{2 \underline{\mu}}{\eta_{2}^{2}} \frac{\lambda_{2}}{\lambda_{1}+\lambda_{2}} .
$$

Solving for $\rho_{2}$ and $\lambda_{1}$ in Eq. 42 and in the first equation of Eq. (43), it is found that

$$
\rho_{2}=\frac{\underline{\mu}}{\rho_{1}},
$$

and

$$
\lambda_{1}=\frac{\eta_{1}^{2} \underline{p}_{1}}{\eta_{2}^{2} \underline{p}_{2}} \lambda_{2}=\frac{\underline{u}}{1-\underline{u}} \lambda_{2},
$$

where $u:=\mathbb{E}\{U\}$ and use was made of Eqs. (40)-411). The idea in this final step is to define the parameters $\rho_{2}$ and $\lambda_{1}$ through Eqs. (44) and 45) (recall that $\left\{\eta_{k}\right\}_{k=1}^{2}$ and $\left\{\underline{p}_{k}\right\}_{k=1}^{2}$ are known from Step 1), and to consider $\rho_{1}$ and $\lambda_{2}$ as the only hyperparameters to be calibrated. For this purpose, let us introduce the cost function $\mathcal{K}$ defined as

$$
\mathcal{K}(x, y):=\sum_{k=1}^{N}\left(\varsigma_{\Sigma}\left(v_{k}^{\exp } ; x, y\right)-\varsigma_{k}^{\exp }\right)^{2},
$$

where $\varsigma_{\Sigma}\left(v_{k}^{\exp } ; x, y\right)$ is the standard deviation of the stress $\Sigma$ at stretch $v_{k}^{\exp }$ (which can be estimated by using the closed-form expressions provided at the end of section 3.2.1, with

$$
\mu \sim \mathrm{G}\left(x, \frac{\mu}{x}\right),
$$

and

$$
U \sim \operatorname{Dir}\left(\frac{\eta_{1}^{2} \underline{p}_{1}}{\eta_{2}^{2} \underline{p}_{2}} y, y\right) .
$$

The hyperparameters $\rho_{1}$ and $\lambda_{2}$ are then defined as the solution of the minimization problem:

$$
\left(\rho_{1}, \lambda_{2}\right):=\underset{(x, y) \in] 0,+\infty[\times] 1,+\infty[}{\operatorname{argmin}} \mathcal{K}(x, y) .
$$

In this work, the optimization problems defined by Eq. (36) and Eq. (49) are solved by using the Matlab function fmincon (with an sqp algorithm) for a set of 1,000 initial guesses determined by Latin Hypercube sampling. Here, the randomization of the starting points aims at reducing in part the impact of the local optimization technique.

\subsection{Discussion}

A number of remarks regarding the calibration strategy are relevant at this point. First of all, the generalization of the calibration method to arbitrary orders shows that the dimension of the problem related to the calibration of $\rho_{1}$ and $\lambda_{n_{p}}$ (which corresponds to $\lambda_{2}$ in the previous section) is, indeed, independent of the values of $m$ and $n$. As a consequence, the methodology does not suffer from a curse of dimensionality and turns out to be very robust in that sense.

Secondly, and while the set of model parameters can be shown to be unique when the constraint equations (given by Eqs. (14), (15), (20) and (21) ) can be all estimated from 
iments, considering the mean and the variability of the stress response does not allow the hyperparameters to be uniquely defined. In fact, it should be observed that the random stress involves nonlinear functions of $\mu$ and $U$. Therefore, a given variability in the random stress equivalently be generated by different combinations of fluctuations in $\mu$ and $U$ (see Eq. (32), (33), (34) and (35)). Note that issues related to (non)uniqueness are also encountered in a deterministic context (which is relevant to the identification of $\left(\eta_{1}, \eta_{2}\right)$ and $\left(\underline{p}_{1}, \underline{p}_{2}\right)$ within Step 1); ee the discussion in 28 for instance.

Additionally, the choice of the hyperparameters to be identified within Step 2 turns out to be arbitrary, so that the associated optimization problem may alternatively be formulated by selecting any couple $\left(\rho_{k}, \lambda_{\ell}\right)$ for $1 \leqslant k \leqslant 2$ and $1 \leqslant \ell \leqslant 2$

Finally, it should be noted that Eq. 45 can be used in order to investigate the sensitivity of the cost function $\mathcal{K}$ (see Eq. (46)) with respect to the second design variable. Indeed, the coefficient of variation $\delta_{U}$ of $U$ is given by

$$
\delta_{U}=\sqrt{\frac{\lambda_{2}}{\lambda_{1}\left(\lambda_{1}+\lambda_{2}+1\right)}},
$$

with

$$
\lambda_{1}=\frac{\underline{u}}{1-\underline{u}} \lambda_{2}
$$

When the mean value $\underline{u}$ (which can be estimated within Step 1 by using Eq. (40) for example) takes small positive values (it is recalled that $0<U<1$ almost surely), one has $\lambda_{1} \ll \lambda_{2}$ and $U$ exhibits large statistical fluctuations. Similarly, it is seen that $\lambda_{1} \gg \lambda_{2}$ when $\underline{u}$ is close to 1 , in which case the fluctuations of $U$ may become very small. In both cases, the coefficient of variation $\delta_{U}$ quickly reaches a plateau as $\lambda_{2}$ becomes larger, and this behavior may decrease the robustness of the formulation within Step 2.

\section{Applications to soft biological tissues}

In this section, the stochastic stored energy functions (with $m=n=1$ ) and the identification methodology are applied in order to model the variability exhibited by various soft biological tissues, including brain and liver tissues, as well as spinal cord white matter.

\subsection{Brain tissue}

In this first application, we address the modeling of the variability exhibited by brain tissues. Toward this aim, we consider the experimental results provided in [17. The $\mathrm{T}^{345}$ for strains ranging up to $50 \%$ and for strain rates $\dot{\varepsilon} \in$ $\{0.5,5,30\} \mathrm{s}^{-1}$. Below, we address the calibration task in unconfined compression for low and medium strain rates. Experimental data are given for white and gray matter, ${ }^{350}$
The optimal mean parameters and exponents $(\boldsymbol{p}, \boldsymbol{\eta})$, computed within Step 1 (see section 3.2), are reported in Tables 1 and 2 for the compression tests at different strain rates.

\begin{tabular}{|c|c|c|}
\hline$\dot{\varepsilon}$ & $0.5 \mathrm{~s}^{-1}$ & $5 \mathrm{~s}^{-1}$ \\
\hline$\underline{p}_{1}(\mathrm{kPa})$ & 0.1467 & 0.1881 \\
$\underline{p}_{2}(\mathrm{kPa})$ & 0.0457 & 0.7823 \\
$\eta_{1}$ & 5.5945 & 4.5907 \\
$\eta_{2}$ & 1.991 & 1.5 \\
$\underline{\mu}(\mathrm{kPa})$ & 2.3863 & 2.3863 \\
$\lambda_{1}$ & 253.5375 & 22.461 \\
$\lambda_{2}$ & 9.9982 & 9.9732 \\
$\rho_{1}$ & 2.3679 & 6.6398 \\
$\rho_{2}$ & 1.0078 & 0.4311 \\
\hline
\end{tabular}

Table 1: calibrated parameters (gray matter in compression).

\begin{tabular}{|c|c|c|}
\hline$\dot{\varepsilon}$ & $0.5 \mathrm{~s}^{-1}$ & $5 \mathrm{~s}^{-1}$ \\
\hline$\underline{p}_{1}(\mathrm{kPa})$ & 1.1131 & 1.4720 \\
$\underline{p}_{2}(\mathrm{kPa})$ & 1.1120 & 0.4897 \\
$\eta_{1}$ & 2 & 2.6185 \\
$\eta_{2}$ & 1.5 & 1.5 \\
$\underline{\mu}(\mathrm{kPa})$ & 3.4772 & 5.5973 \\
$\lambda_{1}$ & 3.2290 & 91.5357 \\
$\lambda_{2}$ & 1.8146 & 9.9919 \\
$\rho_{1}$ & 33.2721 & 6.6819 \\
$\rho_{2}$ & 0.1045 & 0.8377 \\
\hline
\end{tabular}

Table 2: calibrated parameters (white matter in compression)

It is seen that the inequality constraints raised by the polyconvexity and coerciveness properties (see Eqs. (9a)(9c)) are fulfilled, so that the mean model is admissible. Regarding the computation of the Lagrange multipliers $\rho_{1}$ and $\lambda_{2}$, performed within the second step of the methodology, the algorithm is found to converge in a few iterations, regardless of the initial guess. The mean and variability of the random stress predicted by the calibrated stochastic model are quantitatively compared with experimental data in Figs. 1, 2, 3 and 4, where the confidence interval at $95 \%$ is also reported.

It can be observed that the first-order stochastic stored energy function can reproduce the experimental results very well for both gray and white matters at strain rates $\dot{\varepsilon} \in\{0.5,5,30\} \mathrm{s}^{-1}$. 


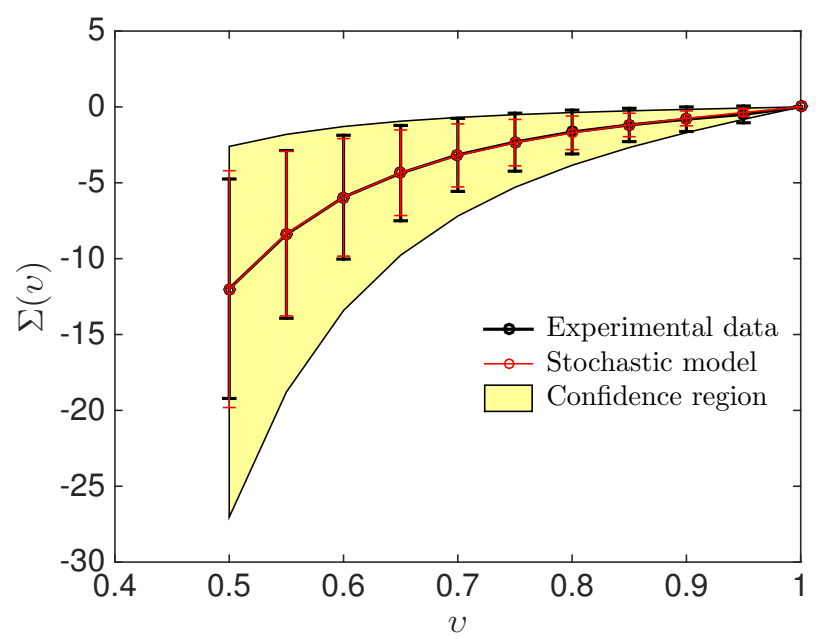

Figure 1: Graph of $v \mapsto \underline{\Sigma}(v)(\mathrm{kPa})$ and $v \mapsto \varsigma_{\Sigma}(v)(\mathrm{kPa})$ for gray matter tissues in unconfined compression at $\dot{\varepsilon}=0.5 \mathrm{~s}^{-1}$. Black lines: experimental data extracted from [17]. Red lines: calibrated stochastic model. Yellow area: 95\% confidence region.

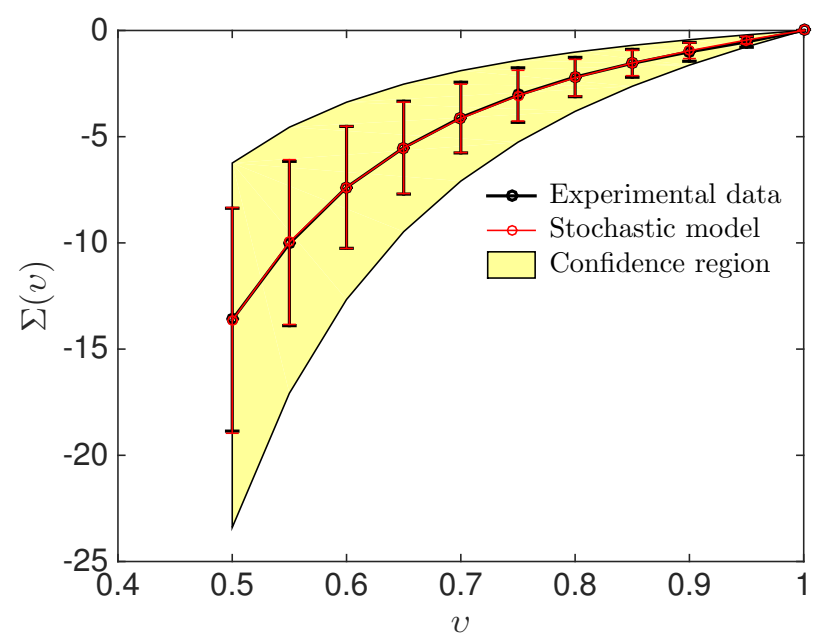

Figure 2: Graph of $v \mapsto \underline{\Sigma}(v)(\mathrm{kPa})$ and $v \mapsto \varsigma_{\Sigma}(v)(\mathrm{kPa})$ for gray matter tissues in unconfined compression at $\dot{\varepsilon}=5 \mathrm{~s}^{-1}$. Black lines: experimental data extracted from [17]. Red lines: calibrated stochastic model. Yellow area: $95 \%$ confidence region.

\subsection{Liver tissue}

365

As a second example, we now turn to the modeling of bovine liver tissue. Specifically, the statistical results are extracted from 33, in which uniaxial compression tests were performed at a $0.01 \mathrm{~s}^{-1}$ strain rate. It should be noted that the (deterministic) fitting in the above reference was obtained by using an exponential-type stored $_{370}$ energy function. The calibrated parameters are listed in Table 3. A qualitative comparison between the statistical properties estimated by means of Monte Carlo simulations with the calibrated stochastic model and those computed from the experiments can be visualized in Fig. 5. Similarly ${ }_{375}$ to the case of brain tissues, it is seen that the probabilistic model and the identification strategy allows the data to be

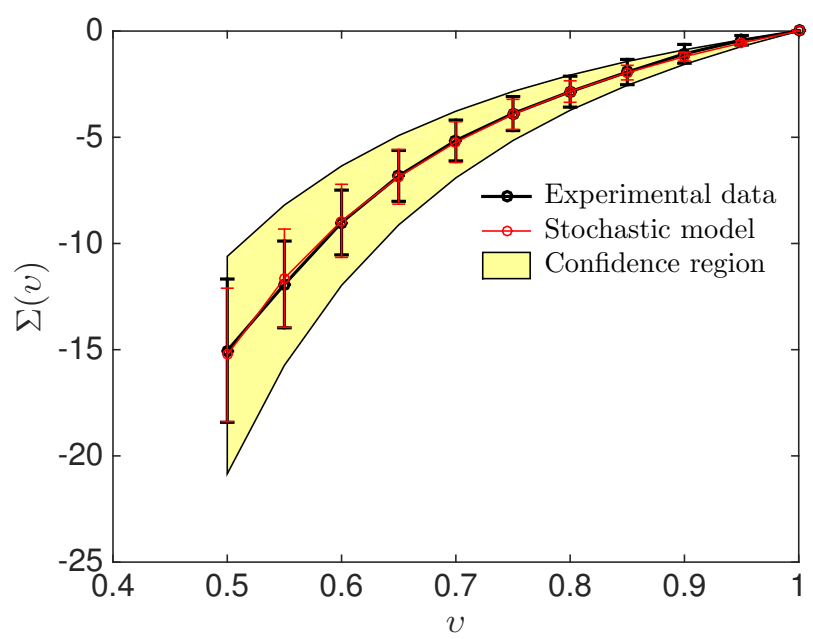

Figure 3: Graph of $v \mapsto \underline{\Sigma}(v)(\mathrm{kPa})$ and $v \mapsto \varsigma_{\Sigma}(v)(\mathrm{kPa})$ for white matter tissues in unconfined compression at $\dot{\varepsilon}=0.5 \mathrm{~s}^{-1}$. Black lines: experimental data extracted from [17]. Red lines: calibrated stochastic model. Yellow area: 95\% confidence region.

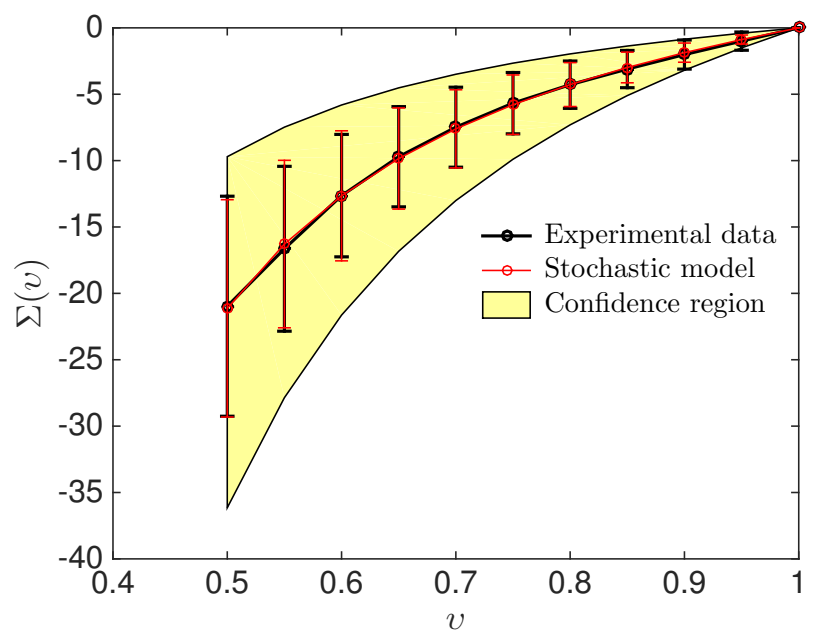

Figure 4: Graph of $v \mapsto \underline{\Sigma}(v)(\mathrm{kPa})$ and $v \mapsto \varsigma_{\Sigma}(v)(\mathrm{kPa})$ for white matter tissues in unconfined compression at $\dot{\varepsilon}=5 \mathrm{~s}^{-1}$. Black lines: experimental data extracted from 17. Red lines: calibrated stochastic model. Yellow area: 95\% confidence region.

reproduced with a reasonably high level of accuracy, hence showing the relevance of the overall methodology.

\subsection{Spinal cord white matter}

In this final application, we address the modeling of the variability exhibited by porcine spinal cord white matter under unconfined compression. Experimental data can be found in 39], where the mechanics of spinal cord injury and its finite element implementation are investigated. The database is composed of 104 independent samples extracted from Yorkshire pigs. Unconfined compression tests were performed for strains ranging up to $40 \%$ and for a strain rate $\dot{\varepsilon} \in\{0.005,0.05,0.5\} \mathrm{s}^{-1}$. The calibrated parameters 


\begin{tabular}{|c|c|}
\hline$\dot{\varepsilon}$ & $0.01 \mathrm{~s}^{-1}$ \\
\hline$\underline{p}_{1}(\mathrm{kPa})$ & 0.1886 \\
$\underline{p}_{2}(\mathrm{kPa})$ & 0.005 \\
$\eta_{1}$ & 2.1783 \\
$\eta_{2}$ & 12.0502 \\
$\underline{\mu}(\mathrm{kPa})$ & 0.8088 \\
$\lambda_{1}$ & 314.6755 \\
$\lambda_{2}$ & 254.251 \\
$\rho_{1}$ & 591.7831 \\
$\rho_{2}$ & 0.0014 \\
\hline
\end{tabular}

Table 3: calibrated parameters (liver tissues in compression).

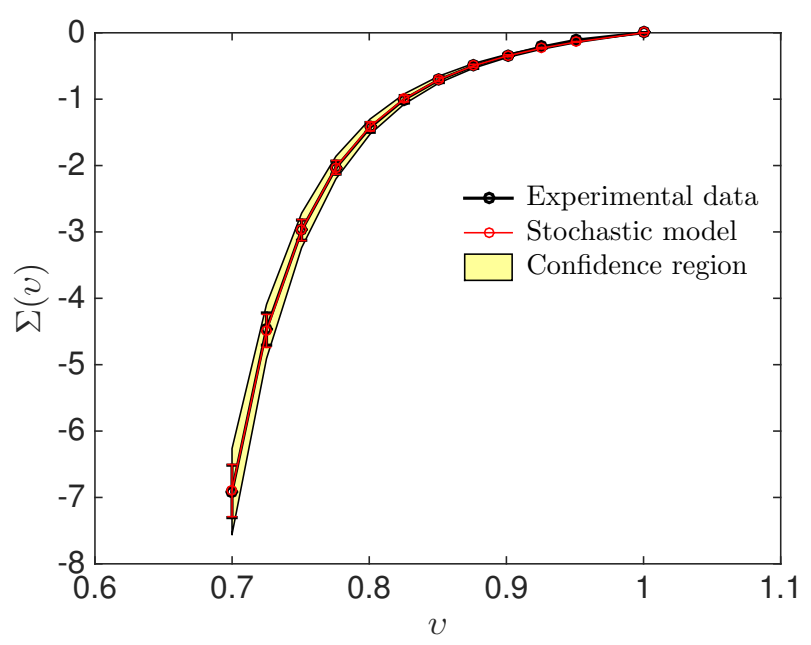

Figure 5: Graph of $v \mapsto \Sigma(v)(\mathrm{kPa})$ and $v \mapsto \varsigma_{\Sigma}(v)(\mathrm{kPa})$ for liver tissues in uniaxial compression at $\dot{\varepsilon}=0.01 \mathrm{~s}^{-1}$. Black lines: experimental data extracted from 34. Red lines: calibrated stochastic model. Yellow area: 95\% confidence region.

are listed in Table 4 and the predicted results are compared with the experimental ones in Figs. 6, 7 and 8.

A very good agreement is again observed between the 395 predictions of the stochastic model and the experimental results.

\section{Conclusion}

In this paper, we have addressed the construction and inverse identification of stochastic constitutive models for hyperelastic biological tissues. The approach builds upon information theory, which essentially allows the constraints related to existence theorems in nonlinear elasticity to be taken into account. This ingredient is key to deriving a stochastic constitutive law that is both physically sound and mathematically consistent. The model can, indeed, be seen as a stochastic extension of the Ogden class of polyconvex stored energy functions, and relies on a low-

\begin{tabular}{|c|c|c|c|}
\hline$\dot{\varepsilon}$ & $0.005 \mathrm{~s}^{-1}$ & $0.05 \mathrm{~s}^{-1}$ & $0.5 \mathrm{~s}^{-1}$ \\
\hline$\underline{p}_{1}(\mathrm{kPa})$ & $1.0003 \times 10^{-10}$ & 0.0102 & 0.0019 \\
$\underline{p}_{2}(\mathrm{kPa})$ & 0.0518 & 0.0060 & 0.0111 \\
$\eta_{1}$ & 2.1306 & 10.3693 & 11.4817 \\
$\eta_{2}$ & 4.5327 & 4.2260 & 5.3651 \\
$\underline{\mu}(\mathrm{kPa})$ & 0.5317 & 0.6048 & 0.2862 \\
$\lambda_{1}$ & $4.2683 \times 10^{-9}$ & 10.2379 & 7.9405 \\
$\lambda_{2}$ & 9.9991 & 1 & 10 \\
$\rho_{1}$ & 25.3357 & 16.6017 & 23.7158 \\
$\rho_{2}$ & 0.0210 & 0.0364 & 0.0121 \\
\hline
\end{tabular}

Table 4: calibrated parameters (spinal white matter in compression).

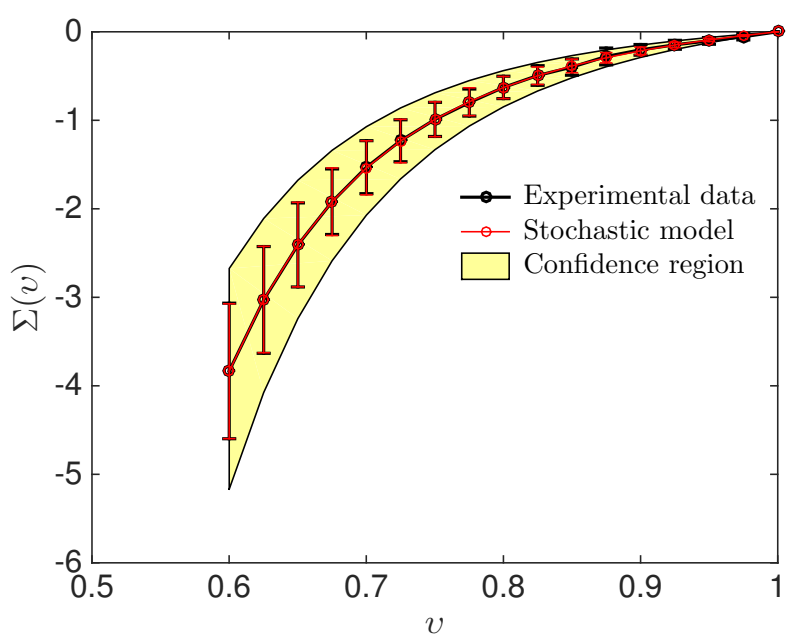

Figure 6: Graph of $v \mapsto \underline{\Sigma}(v)(\mathrm{kPa})$ and $v \mapsto \varsigma_{\Sigma}(v)(\mathrm{kPa})$ for spinal cord white matter in unconfined compression at $\dot{\varepsilon}=0.005 \mathrm{~s}^{-1}$. Black lines: experimental data extracted from 39. Red lines: calibrated stochastic model. Yellow area: 95\% confidence region.

dimensional parametrization that makes the calibration procedure well-posed. The latter was specifically discussed through a sequential strategy. The probabilistic framework was finally applied on different living tissues. It was shown that the model allows the experimental mean behavior and variability to be accurately reproduced at a given strain rate, hence paving the way for a potential generalization accommodating viscous effects. Such a generalization will be the subject of future works.

\section{Appendix A. Maximum Entropy principle}

\section{Appendix A.1. General derivations}

Let $\boldsymbol{A}$ be a vector-valued random variable defined by a probability density function $p_{\boldsymbol{A}}$. We assume that the probability density function $p_{\boldsymbol{A}}$, defined from $\mathbb{R}^{n}$ into $\mathbb{R}^{+}$, has a support which is denoted by $\mathcal{S}_{\boldsymbol{A}}$ (hence, $p_{\boldsymbol{A}}(\boldsymbol{a})=0$ for $\boldsymbol{a} \notin \mathcal{S}_{\boldsymbol{A}}$ ), with $\mathcal{S}_{\boldsymbol{A}} \subseteq \mathbb{R}^{n}$. It is assumed that some 


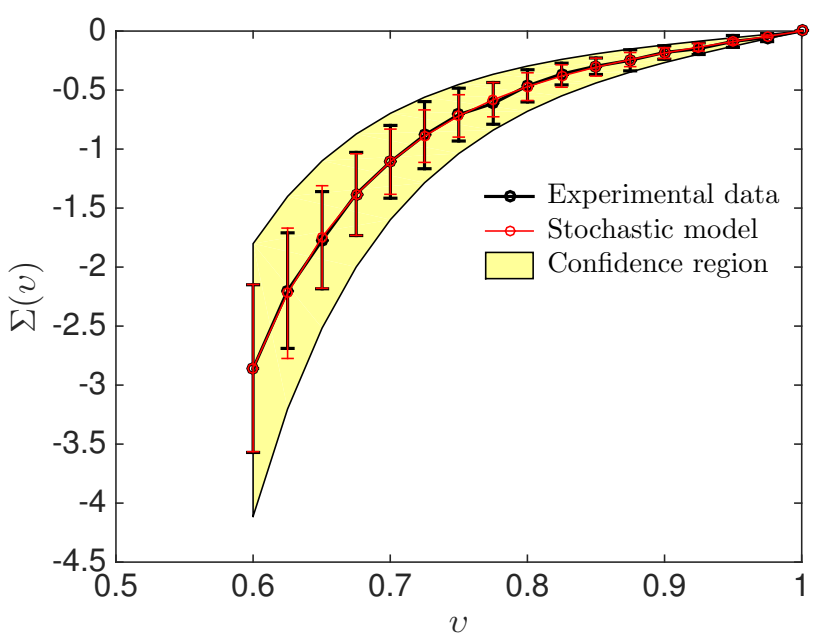

Figure 7: Graph of $v \mapsto \Sigma(v)(\mathrm{kPa})$ and $v \mapsto \varsigma_{\Sigma}(v)(\mathrm{kPa})$ for spinal cord white matter in unconfined compression at $\dot{\varepsilon}=0.05 \mathrm{~s}^{-1}$. Black lines: experimental data extracted from 39]. Red lines: calibrated stochastic model. Yellow area: 95\% confidence region.

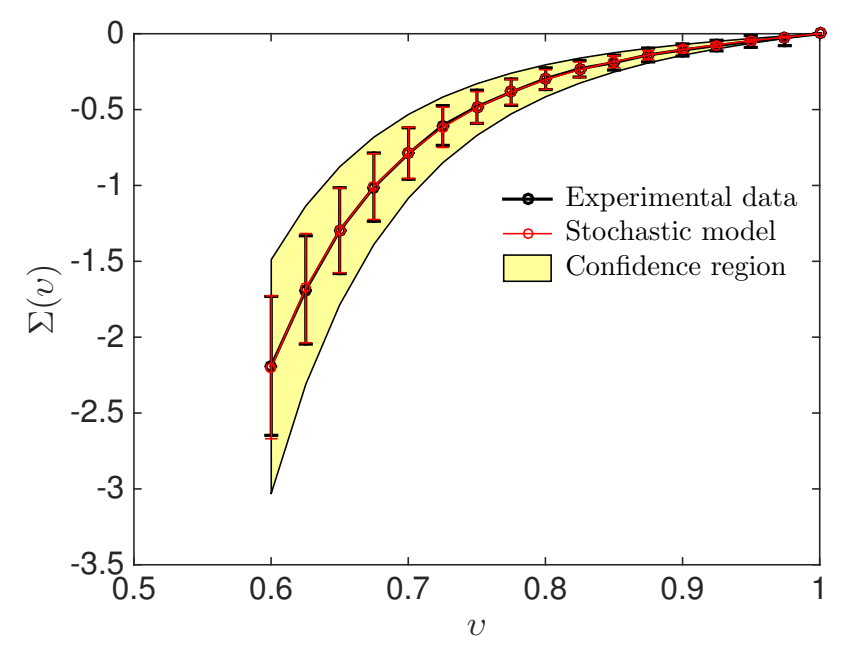

Figure 8: Graph of $v \mapsto \Sigma(v)(\mathrm{kPa})$ and $v \mapsto \varsigma_{\Sigma}(v)(\mathrm{kPa})$ for spinal cord white matter in unconfined compression at $\dot{\varepsilon}=0.5 \mathrm{~s}^{-1}$. Black lines: experimental data extracted from 39. Red lines: calibrated stochastic model. Yellow area: 95\% confidence region.

objective information is available and can be formalized in the form of a mathematical expectation:

$$
\mathbb{E}\{\boldsymbol{h}(\boldsymbol{A})\}=\boldsymbol{f},
$$

where $\boldsymbol{h}$ is a given measurable mapping from $\mathbb{R}^{n}$ into $\mathbb{R}^{N}$, with $N \geqslant 1$, and $\boldsymbol{f}$ is a given vector in $\mathbb{R}^{N}$. The MaxEnt principle then states that the most objective model, given the above constraints, is given by [16, 15, 36]

$$
p_{\boldsymbol{A}}=\underset{p \in \mathcal{C}_{\boldsymbol{A}}}{\operatorname{argmax}} \mathbb{S}\{p\},
$$

where

$$
\mathbb{S}\{p\}:=-\int_{\mathbb{R}^{n}} p(\boldsymbol{A}) \log (p(\boldsymbol{A})) \mathrm{d} \boldsymbol{a}
$$

is the so-called Shannon's entropy of $p$ and $\mathcal{C}_{\boldsymbol{A}}$ is the set of all probability density functions, with support $\mathcal{S}_{\boldsymbol{A}}$, satisfying the constraints defined by Eq. A.1. In order to solve the above functional optimization problem, a Lagrange multiplier $\boldsymbol{\tau}$ with values in an admissible set $\mathbb{T} \subset \mathbb{R}^{N}$ and associated with Eq. A.1 is introduced. Proceeding with the calculus of variation on the associated Lagrangian (see e.g. [19]), the solution is found to be defined as

$$
p_{\boldsymbol{A}}(\boldsymbol{a})=\mathbb{1}_{\mathcal{S}_{\boldsymbol{A}}}(\boldsymbol{a}) k_{0} \exp \left(-\sum_{j=1}^{N} \tau_{j} h_{j}(\boldsymbol{a})\right),
$$

where $\mathbb{1}_{\mathcal{S}_{\boldsymbol{A}}}$ is the indicator function of $\mathcal{S}_{\boldsymbol{A}}$ and $k_{0}$ is a 405 nor normalization constant such that $\int_{\mathcal{S}_{\boldsymbol{A}}} p_{\boldsymbol{A}}(\boldsymbol{a}) \mathrm{d} \boldsymbol{a}=1$.

\section{Appendix A.2. Application 1: probabilistic modeling of the random shear modulus $\mu$}

It is assumed that the constraints related to the random shear modulus $\mu$ are given by Eqs. (15) and 16 . Hence, one has

$$
\boldsymbol{h}(\mu)=\left[\begin{array}{c}
\mu \\
\log (\mu)
\end{array}\right], \quad \boldsymbol{f}=\left[\begin{array}{c}
\underline{\mu} \\
\nu
\end{array}\right]
$$

with $n=1$ and $N=2$ in this particular case. Let $(1-$ $\left.\tau_{1}\right)$ and $\tau_{2}$ be two Lagrange multipliers associated with the two constraints. Using Eq. A.4, it is found that the probability density function $p_{\mu}$ writes

$$
p_{\llbracket}(\mu)=\mathbb{1}_{\mathbb{R}_{+}^{*}}(\mu) k_{0} \exp \left(-\tau_{2} \mu-\left(1-\tau_{1}\right) \log (\mu)\right) .
$$

Upon using the changes of variables $\rho_{1}=\tau_{1}$ and $\rho_{2}=1 / \tau_{2}$, it is seen that $p_{\mu}$ can be written as

$$
p_{\mu}(\mu)=\mathbb{1}_{\mathbb{R}_{+}^{*}}(\mu) k_{0} \mu^{\rho_{1}-1} \exp \left(-\frac{\mu}{\rho_{2}}\right) .
$$

As a result, the random shear modulus $\mu$ is a Gamma random variable with parameters $\rho_{1}>0$ and $\rho_{2}>0$.

\section{normalized random variable $\boldsymbol{U}$}

The probability density function $p_{\boldsymbol{U}}$ of random variable $\boldsymbol{U}$ is constructed by considering that the only available information is given by Eqs. 20, and 21), with $n=n_{p}-1$, $N=n_{p}$ and

$$
\boldsymbol{h}(\boldsymbol{u})=\left[\begin{array}{c}
\log \left(u_{1}\right) \\
\log \left(u_{2}\right) \\
\vdots \\
\log \left(u_{n_{p}-1}\right) \\
\log \left(1-\sum_{k=1}^{n_{p}-1} u_{k}\right)
\end{array}\right], \quad \boldsymbol{f}=\left[\begin{array}{c}
\nu_{1} \\
\nu_{2} \\
\vdots \\
\nu_{n_{p}-1} \\
\nu_{n_{p}}
\end{array}\right] .
$$


Let $\left(1-\lambda_{k}\right)$, for $1 \leqslant k \leqslant n_{p}$, be $n_{p}$ Lagrange multipliers associated with the above constraints. Applying the Max-460 Ent principle (see Eq. A.4), it is found that $p_{\boldsymbol{U}}$ takes the form

$$
\begin{gathered}
p_{\boldsymbol{U}}=\mathbb{1}_{\mathcal{S}}(\boldsymbol{u}) k_{0} \exp \left(-\sum_{k=1}^{n_{p}-1}\left(1-\lambda_{k}\right) \log \left(u_{k}\right)\right. \\
\left.-\left(1-\lambda_{n_{p}}\right) \log \left(1-\sum_{k=1}^{n_{p}-1} u_{k}\right)\right),
\end{gathered}
$$

[17] X. Jin, F. ZHU, H. MAO, M. SHEN, AND K. H. YANG, A com-

where the support $\mathcal{S}$ is given by Eq. 19 . After little algebra, it is found that $p_{\boldsymbol{U}}$ reads as

$p_{\boldsymbol{U}}(\boldsymbol{u})=\mathbb{1}_{\mathcal{S}}(\boldsymbol{u}) k_{0}\left(\prod_{k=1}^{n_{p}-1} u_{k}^{\lambda_{k}-1}\right) \times\left(1-\sum_{k=1}^{n_{p}-1} u_{k}\right)^{\lambda_{n_{p}}-1475}$

As a result, see e.g. [18, random variable $\boldsymbol{U}$ follows $\mathrm{a}^{480}$ Dirichlet distribution with parameters $\left(\lambda_{1}, \lambda_{2}, \ldots, \lambda_{n_{p}}\right)$. Note that the Dirichlet distribution corresponds to a clas-

sical Beta distribution for $n_{p}=2$.

\section{References}

[1] J. M. BALL, Convexity conditions and existence theorems in nonlinear elasticity, Archive for rational mechanics and Analy- ${ }_{490}$ sis, 63 (1977), pp. 337-403.

[2] — Some recent developments in nonlinear elasticity and its applications to materials science, in Nonlinear Mathematics and its Applications, P. J. Aston, ed., Cambridge University Press, Cambridge, 2006, pp. 93-119.

[3] D. Balzani, P. NefF, J. Schröder, and G. A. Holzapfel, A polyconvex framework for soft biological tissues. adjustment to experimental data, International journal of solids and structures, 43 (2006), pp. 6052-6070.

[4] J. Bernado And A. Smith, Bayesian Theory, Wiley, 2000.

[5] G. Chagnon, M. Rebouah, and D. Favier, Hyperelastic energy densities for soft biological tissues: A review, Journal of Elasticity, (2014), pp. 1-32.

[6] C. Chui, E. Kobayashi, X. Chen, T. Hisada, and I. Sakuma, Combined compression and elongation experiments and nonlinear modelling of liver tissue for surgical simulation, Medical and Biological Engineering and Computing, 42 (2004), pp. 787798.

[7] P. G. Ciarlet, Mathematical elasticity: Three-dimensional elasticity, vol. 1, Elsevier, 1993.

[8] L. Devroye, Non-Uniform Random Variate Generation, vol. 1, Springer-Verlag, 1986.

[9] T. C. Gasser, R. W. Ogden, and G. A. Holzapfel, Hyperelastic modelling of arterial layers with distributed collagen fibre orientations, Journal of the royal society interface, $3(2006){ }_{515}$ pp. $15-35$.

[10] A. Gelman, J. Carlin, H. Stern, and D. Rubin, Bayesian Data Analysis, Chapman \& Hall/CRC, 2003.

[11] J. Guilleminot And C. Soize, On the statistical dependence for the components of random elasticity tensors exhibiting material $_{520}$ symmetry properties, Journal of Elasticity, 111 (2013), pp. 109130.

[12] G. A. Holzapfel, T. C. Gasser, and R. W. Ogden, A new constitutive framework for arterial wall mechanics and a comparative study of material models, Journal of elasticity and the $\mathrm{e}_{525}$ physical science of solids, 61 (2000), pp. 1-48.

[13] G. A. Holzapfel and R. W. Ogden, Constitutive modelling of arteries, Proceedings of the Royal Society of London A: Mathematical, Physical and Engineering Sciences, 466 (2010), pp. 1551-1597. prehensive experimental study on material properties of human brain tissue, Journal of Biomechanics, 46 (2013), pp. 2795 2801.

[14] M. Hrapko, J. Van Dommelen, G. Peters, and J. Wismans, Characterisation of the mechanical behaviour of brain tissue in compression and shear, Biorheology, 45 (2008), pp. 663-676.

[15] E. T. JAYNES, Information theory and stastitical mechanics ii, Physical Review, 108 (1957), pp. 171-190.

16] - Information theory and statistical mechanics $i$, Physical Review, 106 (1957), pp. 620-630.

$18]$ N. L. Johnson, S. Kotz, and N. Balakrishnan, Continuous multivariate distributions, volume 1, models and applications, vol. 59, New York: John Wiley \& Sons, 2002

[19] D. G. LuenBerGer, Optimization by vector space methods, John Wiley \& Sons, 1969.

[20] S. Madireddy, B. Sista, And K. Vemaganti, A bayesian approach to selecting hyperelastic constitutive models of soft tissue, Computer Methods in Applied Mechanics and Engineering, 291 (2015), pp. 102-122.

[21] - Bayesian calibration of hyperelastic constitutive models of soft tissue, Journal of the mechanical behavior of biomedical materials, 59 (2016), pp. 108-127.

[22] K. Miller AND K. ChinzeI, Constitutive modelling of brain tissue: experiment and theory, Journal of biomechanics, 30 (1997), pp. 1115-1121.

23] - Mechanical properties of brain tissue in tension, Journal of biomechanics, 35 (2002), pp. 483-490.

[24] C. B. Morrey, Quasi-convexity and the lower semicontinuity of multiple integrals, Pacific J. Math, 2 (1952), pp. 25-53.

[25] S. MÜller, T. QI, AND B. YAN, On a new class of elastic deformations not allowing for cavitation, Annales de l'I.H.P. Analyse non linéaire, 11 (1994), pp. 217-243.

[26] A. Nouy, Identification of multi-modal random variables through mixtures of polynomial chaos expansions, Comptes Rendus Mécanique, 338 (2010), pp. 698 - 703.

[27] R. W. Ogden, Non-Linear Elastic Deformations, Dover Publications, Mineola, New York, 1987.

[28] R. W. Ogden, G. Saccomandi, And I. Sgura, Fitting hyperelastic models to experimental data, Computational Mechanics, 34 (2004), pp. 484-502.

[29] B. Rashid, M. Destrade, And M. D. Gilchrist, Mechanical characterization of brain tissue in compression at dynamic strain rates, Journal of the mechanical behavior of biomedical materials, 10 (2012), pp. 23-38.

[30] - Mechanical characterization of brain tissue in simple shear at dynamic strain rates, Journal of the mechanical behavior of biomedical materials, 28 (2013), pp. 71-85.

[31] _ Mechanical characterization of brain tissue in tension at dynamic strain rates, Journal of the mechanical behavior of biomedical materials, 33 (2014), pp. 43-54.

[32] B. Rashid, M. Gilchrist, And M. Destrade, Experimental characterisation of neural tissue at collision speeds, in International Research Council on Biomechanics of Injury Conference (IRCOBI 2012), International Research Council on Biomechanics of Injury (IRCOBI), 2012.

[33] E. RoAN AND K. VEMAGANTI, The nonlinear material properties of liver tissue determined from no-slip uniaxial compression experiments, Journal of biomechanical engineering, 129 (2007), pp. $450-456$.

[34] - Strain rate-dependent viscohyperelastic constitutive modeling of bovine liver tissue, Medical \& biological engineering \& computing, 49 (2011), pp. 497-506.

[35] I. Sakuma, Y. Nishimura, C. K. Chui, E. Kobayashi, H. InADA, X. Chen, AND T. HisAdA, In vitro measurement of mechanical properties of liver tissue under compression and elongation using a new test piece holding method with surgical glue, in Surgery Simulation and Soft Tissue Modeling, Springer, 2003, pp. 284-292.

[36] C. E. Shannon, A mathematical theory of communication, Bell System Technical Journal, 27 (1948), pp. 379-423/623-659. 
[37] C. SoIze, A computational inverse method for identification of non-gaussian random fields using the bayesian approach in very high dimension, Computer Methods in Applied Mechanics and Engineering, 200 (2011), pp. 3083 - 3099. vector, SIAM/ASA J. Uncertainty Quantification, 3 (2015), pp. $34-60$.

[39] C. J. Sparrey and T. M. Keaveny, Compression behavior of porcine spinal cord white matter, Journal of biomechanics, 44 (2011), pp. 1078-1082.

[40] B. Staber and J. Guilleminot, Stochastic modeling of a class of stored energy functions for incompressible hyperelastic materials with uncertainties, Comptes Rendus Mécanique, 343 (2015), pp. 503-514.

[41] _ Stochastic modeling of the ogden class of stored energy functions for hyperelastic materials: the compressible case, ZAMM - Journal of Applied Mathematics and Mechanics, (2016). Accepted for publication.

$42]$ S. Umale, C. Deck, N. Bourdet, P. Dhumane, L. Soler, J. Marescaux, and R. Willinger, Experimental mechanical characterization of abdominal organs: liver, kidney 83 spleen, Journal of the mechanical behavior of biomedical materials, 17 (2013), pp. 22-33.

[43] F. Velardi, F. Fraternali, and M. Angelillo, Anisotropic constitutive equations and experimental tensile behavior of brain tissue, Biomechanics and modeling in mechanobiology, 5 (2006), pp. 53-61. 